\title{
GSFRS: Giant Signal File Random Sampler
}

This paper was downloaded from TechRxiv (https://www.techrxiv.org).

\section{LICENSE}

CC BY 4.0

SUBMISSION DATE / POSTED DATE

$18-07-2021 / 20-07-2021$

CITATION

Pal, Prasanta; Brewer, Judson (2021): GSFRS: Giant Signal File Random Sampler. TechRxiv. Preprint. https://doi.org/10.36227/techrxiv.15000945.v1

$\mathrm{DOI}$

10.36227/techrxiv.15000945.v1 


\title{
GSFRS: Giant Signal File Random Sampler
}

\author{
Prasanta Pal and Judson A. Brewer
}

\begin{abstract}
In the modern world, it is hard to imagine a day without some form of interaction with digital data. Real world data originating from signal generating transducers or communication channels are often recorded as streams of data samples separated by time stamps, sample counters or simply data record delimiter e.g. newline $(\backslash n)$, comma (,) etc. Sampling is the basis of statistical estimation from any data source containing signal records. The process of random sampling has been in practice since time immemorial. However, rapid scale of data generation processes working in tandem with of computing infrastructures , the volume of data is getting quite unmanageably large in nearly every discipline of science. On the other hand, mere volume of data is of no consequence if we can't extract effective intelligence out of it on an "on demand" basis. Of particular interest is the case where data is stored in a file as a record separated by newline(or any other delimiter) character. When the number of records in the file is greater than a threshold, random sampling is a formidable task. It is nearly impossible to pragmatically load the entire file in the computer memory or even if theoretically possible, the time it takes to load the data in its entirety from natural data sources can be overwhelmingly long or often unnecessary! We can strategically bypass these problems by carefully designing a data interface tool such that any part of a given file can be instantly accessed for random sampling or other kinds of processing tasks by loading only the necessary parts of the data. With this goal, we created a novel, portable and highly efficient rapid data access tool named GSFRS: Giant Signal File Random Sampler, written in modern $\mathrm{C}++_{+}$language to enable near real-time access to any part of an arbitrarily large sized data file that is almost independent of the file size for all practical scenarios. Also, big-data processing would become relatively commonplace and cost effective even in commodity hardwares once the indices are made available through its indexing protocol. This capability would potentially revolutionize the way we gather intelligence from files containing large samples. Adaptation of GSFRS at the source level of various data generators, processing times and energy footprints of various computations can be dramatically reduced.
\end{abstract}

Index Terms-Big data, Random sampling, Data parsing, File handling, Data indexing, Map reduce, Signal processing, $\mathrm{C}_{++}$, CPP

\section{INTRODUCTION}

C Tatistical estimation of the nature of measured digital $\mathcal{S}$ signal is at the heart of signal processing tasks associated with large data sets. Behavior of large-scale systems are understood primarily through strategic random sampling techniques [1], [2], [3]. The entire machinery around widely

Prasanta Pal is with the Brown Mindfulness Center, School of Public Health and Department of Epedemiology, Brown University, RI, 02912 USA e-mail: (prasanta.pal@gmail.com).

Judson A. Brewer is with the Brown Mindfulness Center, School of Public Health and Department Psychiatry, Behavior and Social Sciences, Brown University

Manuscript received June 10, 2021; revised June 10, 2021. popular Monte-Carlo (MC) methods [4], [5], |6], |7], [8] hinges on the foundation of random sampling of data. MC is a relatively simple task for small data sets. However, with the stupendous growth in size of data sources, such a task is almost formidable because of the lack of random access to various parts of data. As per current standards, the only non-trivial way to read a large data file is by first opening the file-stream 1 followed by reading sequentially in a byte by byte fashion. Even with moderate file sizes, important but expensive computations like autocorrelation $(A C F)$ [9], [10], [10], [11] or mean-square-displacement (MSD) [7], [12], [13] functions with multiple time-lags $\left(\tau_{k \in 1,2, . . n}\right)$ would practically be impossible without having random access to every record of a large data file. Because of the above reasons, we almost never observe calculations involving $a u$ tocorrelation with large $\tau$ values in the published literature despite $A C F, M S D$ being very important measures for time series analysis. To evaluate expensive computations like the above function at multiple discrete time lags $\left(\tau_{k}\right)$, we have to re-read the file many times corresponding to each $\tau_{k}$ ! Also, as microprocessor architectures become increasingly multi-core in nature [14], [15], [16], optimal utilization of multiple cores and available thread resources are impossible to achieve without fundamentally re-engineering the problem of asynchronous random access using modern multithreaded architecture [17], [18]. Often, in many realistic scenarios, data records are stored in an ASCII or binary file with each line containing a sample of the data as a time stamp or serial number record. Some common examples are, highdensity EEG data, system logs of digital events, data from financial markets etc. Even though, modern computers are enormously efficient in reading and writing small data segments from storage medium like hard drives, however, due to high quality and high-speed nature of modern real world data generation processes, the size of the data sets are also exponentially growing. So this creates a significant burden in obtaining statistical estimates of these data samples. Traditionally, large databases [19], [20], [21], [22] are utilized to get instant access to various parts of stored data. The rapid rise of custom, niche databases [23], [24] to address problem specific targeted access to large datasets is testament to the importance and need of having capabilities around customizable database technologies. A lot of big-data driven discoveries are enabled by these modern database technologies [25], [26]. However, in research, desktop prototype development, remote processing environments, databases

1. https://en.cppreference.com/w/cpp/io/basic_fstream 
beyond few giga-bytes is often a significantly resource consuming (e.g. infrastructure cost, energy, high speed internet) undertaking. Mobile and remote systems that operate on battery power, constant supply of energy is needed to manage the database infrastructure on the host machine. Even in desktop computing scenarios, often all the processing power of the multi-core processors are not optimally utilized. In order to address these issues, we developed a random data access tool capable sampling data from an arbitrarily large source data file at near clock speed of the host device without ever having to load the entire file in the memory. This tool is highly flexible, with low energy footprint and high portability. Theoretically speaking, GSFRS would work up to approximately 18 exa-bytes of storage space for 64 bit operating system architectures. Parallel computations on various parts of the data from independent processes would be a very natural practice as the need to perform serial reading from the start of the file would be completely unnecessary.

\section{Method}

\subsection{Assumptions, symbols and definitions}

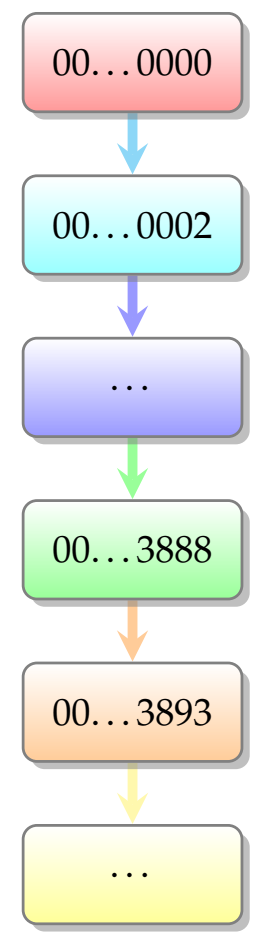

Fig. 1: Schematic diagram to illustrate the structure of the index file. Consecutive index blocks each of size $\mathcal{N}^{\mathcal{F} . \text { idx }}$ contain the ordered sequence of indices for $\mathcal{D}^{c}$ as it appears in $\mathcal{F}_{i n}$.

The file type of the data source is assumed to be in ASCII format. The input file containing signal data is denoted as $\mathcal{F}_{i n}$. The number of characters in $\mathcal{F}_{i n}$ is denoted as $\mathcal{N}^{\mathcal{F}_{i n}}$. For a given character $c$ when used as a data sample delimiter (or separator) is denoted as $\mathcal{D}^{c}$. A delimiter separated data sample $\mathbb{S}_{k}$ is defined as the sequence of characters between $(k-1)^{t h}$ and $k^{t h}$ instance of $\mathcal{D}^{c}$ in $\mathcal{F}_{i n}$ e.g. for newline as delimiter it is the sequence of characters in the $k^{t h}$ line. Index of a character is the byte location of that character in a given file. The underlying operating system has $N=64$ bit architecture ${ }^{2}$ Index file denoted as $\mathcal{F}_{\text {.idx }}$ is the file containing the indices of the $\mathcal{D}^{c}$. We make a convention that, the first line of the index file contains the meta information about the file and the rest of the file starting from the second line contains the indices.

\subsection{Algorithm, dependencies and relationships}

Three important $C++$ native functions [27], [28], [29] are the key drivers behind the functionalities of GSFRS. (a) seekg, (b) tellg and (c) read ${ }^{3}$ The seekg function sets the current open stream position to a specific target byte location on the file-stream whereas, tellg returns the current byte location of the open file-stream and read, reads a set number of characters from the current byte location to a temporary buffer array. GSFRS works in a two-step fashion, (a) indexing of $\mathcal{F}_{i n}$ to produce $\mathcal{F}_{\text {.idx }}$ and (b) sampling or parsing of $k^{t h}$ instance of $\mathbb{S}_{k}$. The primary goal of step (a) is to find and store the indices of $\mathcal{D}^{c}$ in an index file corresponding to $\mathcal{F}_{i n}$. In order to do so in an efficient manner, we took advantage of the inherent multi-threaded architecture of modern microprocessors [30], [31]. A schematic representation of the index structure as recorded in $\mathcal{F}_{\text {.idx }}$ is presented in Fig. 1 A representative snapshot of a typical $\mathcal{F}_{\text {.idx }}$ is shown in Fig. 2. The quantative details of the files are annotated within the representative figure. In order to execute multithreaded processing, $\mathcal{F}_{i n}$ is virtually divided into $\mathcal{N}^{\mathcal{B}}$ datablocks as defined in Eq. 1 so that each datablock can be delegated to an individual thread made available through the 童害.

$$
\mathcal{N}^{\mathcal{B}}=\operatorname{ceil}\left(\frac{\mathcal{N}^{\mathcal{F}_{i n}}}{\mathcal{N}^{\text {in }}}\right)
$$

where, ceiling is the standard mathematical ceiling [32 function. $k^{t h}$ data-block is located between $(k-1) \times \mathbf{m}$ and $k \times \mathbf{m}$ byte locations. For each $\mathcal{B}_{k}$ we store the indices in a temporary index file $\mathcal{F}_{\text {temp }}$ created under $/$ tmp ${ }^{4}$ folder location of the host $O S$. Once all the threads complete the individual jobs, all the indices are merged into a single file $\mathcal{F}_{\text {.idx }}$ in order followed by removal of all the instances of $\mathcal{F}_{\text {temp. }}$. This way the memory footprint of the indexing operation is kept under control without significant penalty of efficiency as all the computationally heavey operations are done in the individual thread level. For the purpose of simplicity, we store the indices with base 10 system in $\mathcal{F}_{\text {.idx }}$ in a consecutive fashion in order of appearance in $\mathcal{F}_{i n} . \mathcal{F}_{\text {.idx }}$ is structured such a way that, the number of place holding characters $\boldsymbol{m}$ to store the indices for all addressable space in $N=64$ bit architecture is derived as shown below in Eq. 2

$$
\begin{aligned}
\mathbf{m} & =\operatorname{ceil}\left(N \times \log _{10}(2)\right) \\
& =20
\end{aligned}
$$

2. it is trivial to adapt to any other architecture types

3. or equivalently fread

4. or any other convenient location 
The index location of a given $\mathcal{D}_{k}{ }^{c}$ is encoded with $m$ length string with zero padded at the beginning 5 of the index representation.

$$
\mathcal{F}_{. i d x}=\mathcal{H}_{. i d x}+\mathbb{D}_{. i d x}
$$

For the purpose of simplicity and data structure management, $\mathcal{F}_{\text {.idx }}$ is divided into two parts. (a) header section $\left(\mathcal{H}_{. i d x}\right)$ and $(\mathrm{b})$ data section $\left(\mathbb{D}_{. i d x}\right)$, as descrived in Eq. 3 Access to the header section of the $\mathcal{F}_{\text {.idx }}$ provides with all the necessary meta information about $\mathcal{F}_{i n}$. The data section contains all the indices in order of appearance in $\mathcal{F}_{i n}$.

$$
\mathcal{I}_{k}^{c}=k \times \mathrm{m}
$$

Eq. 4 shows the relationship between an index location for a given sample numver $k$. This information is utilized to query the index for a given sample number. An overview of the basic life-cycle of GSFRS operations (e.g. indexing \& parsing) are described in Figs. 3 \& 4 respectively. Random sampling is meaningful only when the index has been created and stored in the index file corresponding to a given $\mathcal{F}_{i n}$. The software would prompt the user to ask for permission to create $\mathcal{F}_{\text {.idx }}$ if sampling is attempted before creation of the index.

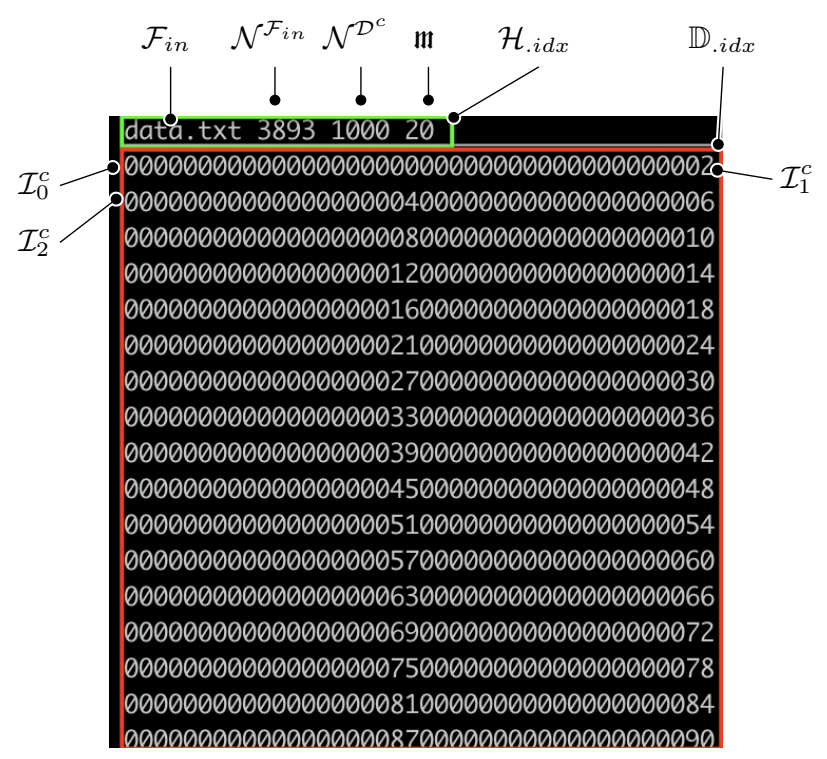

Fig. 2: File structure of a representative index file $\mathcal{F}_{\text {.idx }}=$ data.txt.idx created from $\mathcal{F}_{i n}=$ data.txt having file size $\mathcal{N}^{\mathcal{F}_{\text {in }}}=17979601824$ bytes and number of lines $\mathcal{N}^{\mathcal{D}^{c}}=$ 9963520 lines. The first line on the top is the header section and rest of the file (red rectangle) contains all the indices corresponding to each $\mathcal{D}^{c}$ in order. Note: For brevity, only first few indices are shown here.

\subsection{Indexing}

The key principle behind the rapid data accessibility through GSFRS is the creation of an index file $\mathcal{F}_{\text {.idx }}$ for a given $\mathcal{F}_{\text {in }}$ and $\mathcal{D}^{c}$. One time indexing (OTI) is the the process of creating $\mathcal{F}_{\text {.idx }}$. During indexing process, the

5. e.g. index 123 would is encoded as 00000000000000000123 entire $\mathcal{F}_{i n}$ is read in its entirety 6 by reading only small relevant chunks of $\mathcal{F}_{i n}$ to asynchronously index each instance of $\mathcal{D}^{c}$, starting from beginning to the last character of $\mathcal{F}_{i n}$. The asynchronous operation is facilitated with the help of a thread-pool ( 墔 ) [33], [34] maintained during the entire lifetime of the indexing or sampling operation. At the initialization stage of the software so that computation can be delegated to different threads when necessary, while avoiding expensive thread-creation and deletion operations during runtime. First, we index the data on receipt and then using that index we access parts of the data. The basic step of indexing starts with finding each and every newline character while reading the file from the beginning till the end of the file. At each occurrence of the newline we make note of the index position and store the record in the index file. This record is encoded in an m digit string format where non significant positions are prepended with 0 as placeholders to fill all the remaining non-significant part of $\boldsymbol{m}$ digits.

\subsection{Sampling}

In the context of GSFRS sampling is equivalent to parsing of certain strategic sections of the data from the source. Parsing of $\mathbb{S}_{k}$ is a two step process. (a) reading the index $\mathcal{N}^{\mathcal{I}_{k}^{c}}$ for $k^{\text {th }}$ sample from $\mathcal{F}_{\text {.idx }}$ and (b) reading the sample $\mathbb{S}_{k}$ from $\mathcal{F}_{i n}$ using the corresponding index $\mathcal{N}^{\mathcal{I}_{k}^{c}} \mathrm{ob}-$ tained from step (a). Both the steps involve three importnant operations (A) file opening, (B) finding byte location through seekg and (C) file closing. The seekg function for $\mathcal{F}_{\text {.idx }}$ and $\mathcal{F}_{\text {in }}$ are utilized to obtain the index and sample respectively corresponding to a sample instance. In the context of this work, sampling is equivalent to parsing the $k^{t h}$ segment of delimiter separated character sequence. This effectively boils down to finding the $k^{\text {th }}$ index location $\left(\mathcal{I}_{k}^{c}\right)$ from $\mathcal{F}_{\text {.idx }}$. From Eq. 4 , we get the beginning of the $m$ length index string. After setting the file stream position on that character we read the next $m$ characters followed by converting it to unsigned long long int format such that this number is the index location of the end of $\mathbb{S}_{k}$. In a similar fashion the $(k-1)^{t h}$ index lcoation is obtained from the open stream of $\mathcal{F}_{\text {in }}$ we read all the characters between $\mathcal{N}^{\mathcal{I}_{k-1}^{\mathcal{C}}}$ and $\mathcal{N}^{\mathcal{I}_{k}^{c}}$. The ordered set of these characters is $\mathbb{S}_{k}$. GSFRS's parsing ability relies on the ordered structure based on the location of the $k^{\text {th }}$ instance $\mathcal{D}_{k}{ }^{c}$ of $\mathcal{D}^{c}$ parsed with the help of both. Because every index is stored as a $m$ length string, the location of index corresponding to $\mathbb{S}_{k}$, in $\mathcal{F}_{\text {.idx }}$ can be calculated with the use of a decoding protocol. Parsing is done by accessing the specific index location corresponding the $\mathcal{D}_{k}{ }^{c}$ in $\mathcal{F}_{\text {.idx }}$. Given an index location $\mathcal{I}_{k}^{c}$, the built-in seekg function can have access to that byte-location in $\mathcal{F}_{\text {.idx }}$. Once a byte-location in $\mathcal{F}_{\text {in }}$ is accessed through Eq. 4 it is trivial to to conditionally (or on demand basis) retrieve the set of characters around that location e.g. $\mathbb{S}_{k}$.

\subsection{Data sources with real world \& simulated signal}

In order to normalize time scales of various calculations, we created a set of simulated signal data files

6. the only time 


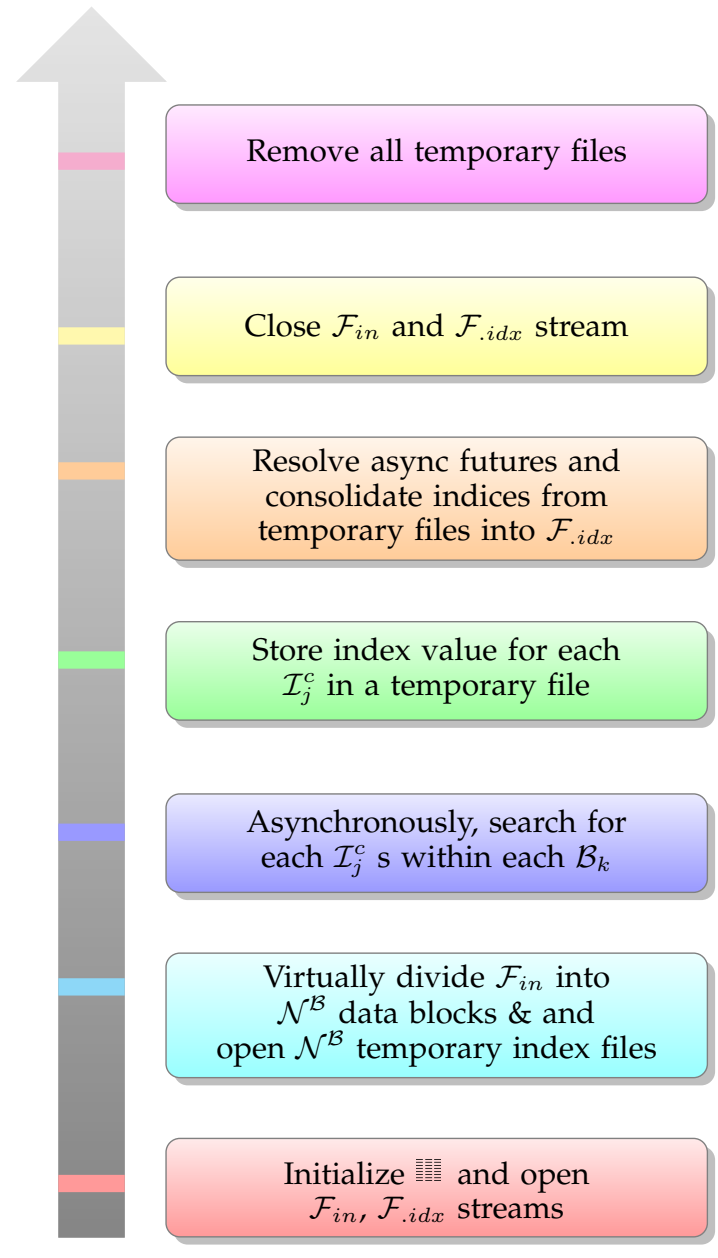

Fig. 3: Indexing steps for a given input file $\mathcal{F}_{i n}$ to create $\mathcal{F}_{\text {.idx }}$

by closely mimicking realistic EEG signal sources but the signal values replaced by fixed width random numbers. For the purpose of testing and calibration we created 7 simulated signal files with 256 columns and $10,100,1000,10000,100000,1000000,10000000$ rows respectively. Each signal element of these simulated files are generated using standard built-in random number generator with fixed number of 6 characters. Calculations related to performance of the software with respect to various relevant parameters were performed on these data sets. In order to perform software validation on a realistic data set, we used a long EEG data file with approximately one hour continuous recording time sampled at $2048 \mathrm{~Hz}$.

\section{Results}

The results are centered around the performance evalutation of GSFRS compared to existing gold standards and clock speed of the host machine. In order to evaluate the performance we used both real world and simulated data. Simulated data was designed and used for calibration and normalization of various relevant time scales whereas, real world experimental data was used to verify the utility of GSFRS in realistic scenarios. For the purpose of uniformity of time scales, all the numerical experiments were performed on the same machines under identical conditions. The machine configuration used for generating the results described

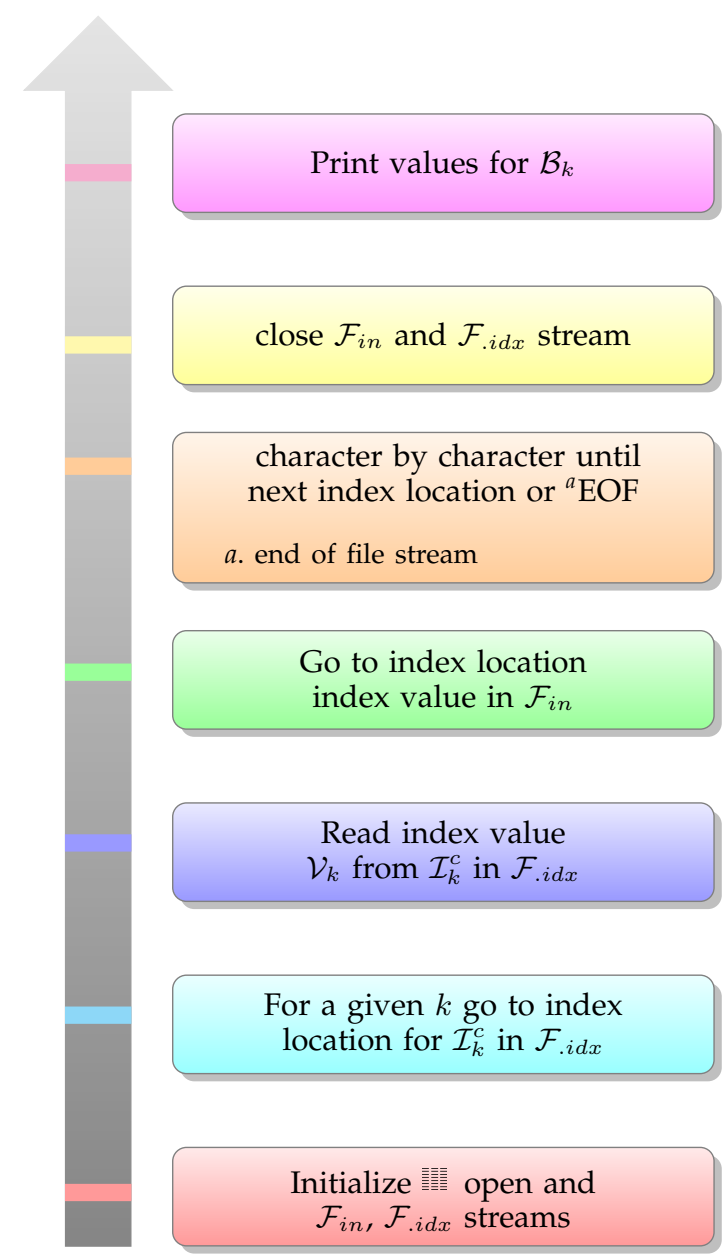

Fig. 4: Parsing steps of a data-block $\mathcal{B}_{k}$ from $\mathcal{F}_{i n}$ by through $\mathcal{F}_{\text {.idx }}$

here is Apple Macbook Pro, 2019, $2.4 \mathrm{GHz}$ 8-Core Intel Core i9. Fig. 5 demonstrates the comparison of sampling time

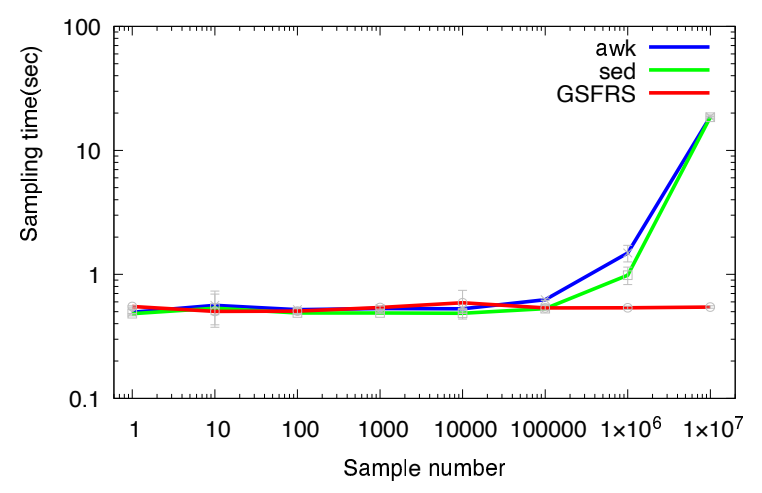

Fig. 5: Comparison of sampling time between GSFRS and other standard tools like awk, sed for various data record locations (e.g. line numbers)

between GSFRS and other standard (unix or linux based) data access tools e.g. $a w k$ and sed. Interpolation lines are drawn to aid visual cue. For each point (e.g. number of samples) on the horizontal axis, 10 sampling instances were performed to calculate the mean and standard deviation of 
the sampling times over those instances. Figs. 6 \& 7 demon-

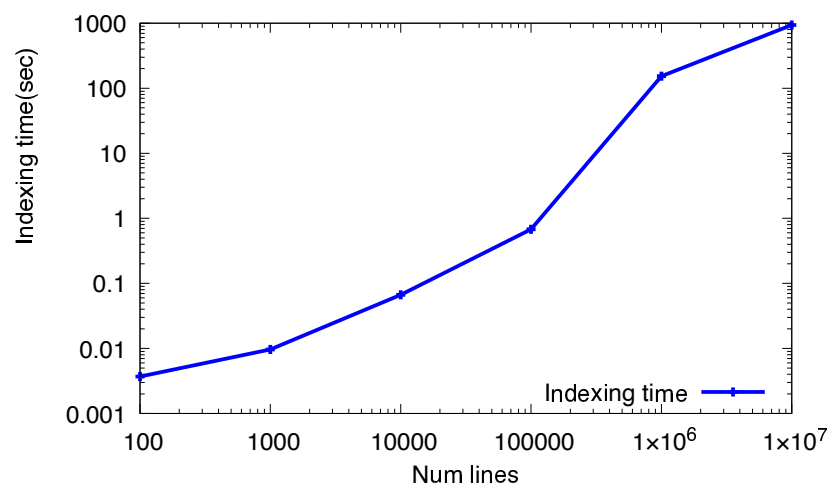

Fig. 6: Indexing time as a function of number of lines in the input file.

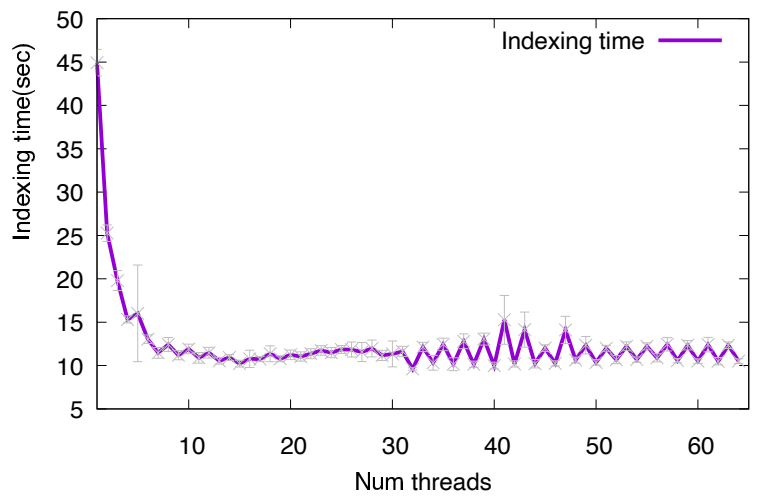

Fig. 7: Indexing time as a function of number of $\mathcal{N}^{\text {int }}$.

strate the indexing time with respect to various relevant parameters. Fig. 6 describes the indexing time (second) as a function of $\mathcal{N}^{\mathcal{D}}$ and Fig. 7 shows the indexing time for a fixed $\mathcal{F}_{i n}$ but varying $\mathcal{N}^{\mathrm{in}}$.

Similarly, Figs. 8 \& 9 demonstrate the sampling time (sec) with respect to various relevant parameters. Fig. 8 describes the sampling time (sec) as a function of the $\mathcal{N}^{c}$ for the simulated signal files described in sec. 2.5 and 9 describes the sampling time (sec) for fixed number of samples $\mathcal{N}^{\mathbb{S}}$ but

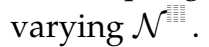

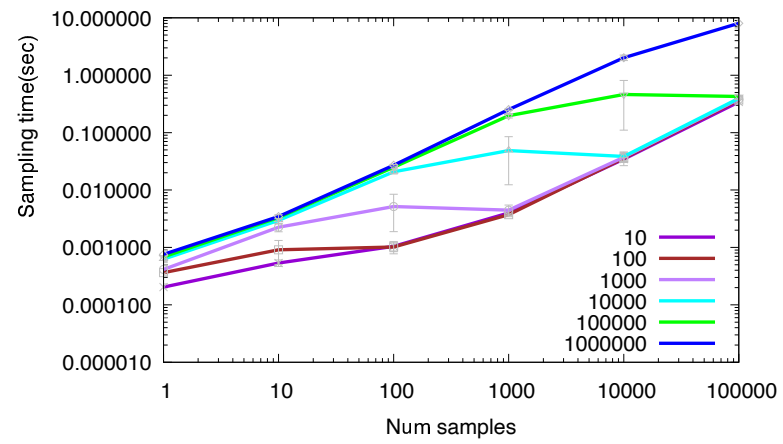

Fig. 8: Sampling time vs number of lines in the $\mathcal{F}_{\text {in }}$

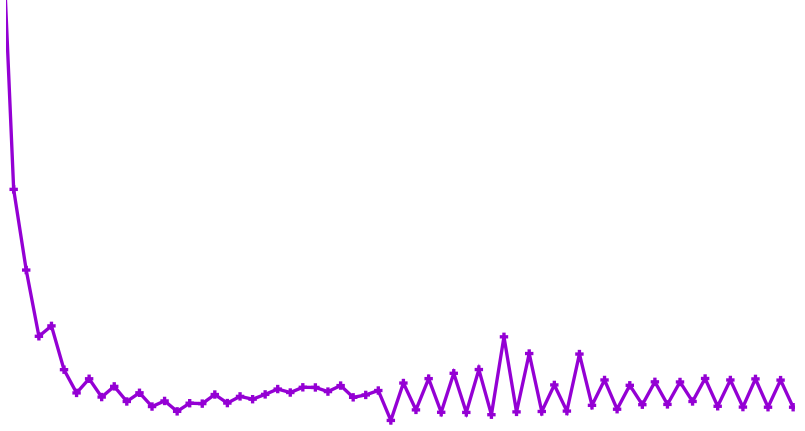

Fig. 9: Sampling time vs number of threads for a a fixed number of samples

\section{Application areas}

Some potential application areas are listed below.

1) Medical devices [35], [36] for on-the-fly data indexing so that diagnostic outcomes can be produced at a rapid pace

2) Off the grid devices [37] requiring low energy footprint for query from large data-sets

3) Edge computing [38 and edge $A I$ applications where low energy footprint and realtime access to collected data is an important criteria for reliable prediction

4) Space applications (e.g. NASA's MARS rovers) [39], [40] performing on-the-fly data analysis

5) Asynchronous query applications on remote devices

6) Real-time systems [41] for multi-threaded access to the same data source

\section{Discussion, Strength ANd Limitations}

GSFRS enables us to have random access and sample any arbitrarily large ASCII file. In the context of a given processing task, only the necessary part of the data can be readily loaded on the memory for processing,without having to access any other part of the source. This is a significant advantage over line-by-line data access method followed in traditional tools like grep, sed, awk etc. where every instance of data reading involves reading the data from the beginning of the file followed by opening of the filestream. These unused data access instances are effectively absorbed by the OTI process as demonstrated in Fig. 6 $O T I$ can be efficiently performed by taking advantage of multi-threading architectures of modern microprocessors. The effect of 彗 based indexing architecture is described in Fig. 7. The indexing time saturates to an optimal value as $\mathcal{N}^{\mathrm{III}} \rightarrow \mathcal{N}^{\text {.II. }}$. The observation, that the performance actually degrades (although slightly), for $\mathcal{N}^{\text {inl }}$ beyond $\mathcal{N}^{\text {may }}$ seem apparently contradictory. One possible explanation is context switching [42 due to computational overload on a given hardware thread. A zig-zag pattern is observed for odd and even number of threads possibly attributed 
to the way the $O S$ and the microprocessor optimizes concurrent access to data streams. Fig. 8 demonstrates that, for relatively small number of samples, the sampling time does not vary much across various file sizes. For moderate range, the differences across file sizes are not very significant although for the file containing 100000 lines, there is a noticeable jump in the sampling time. It is clear that the computational cost 7 gets overall higher as the number of samples increases. In Fig. 9. we observe that the query time is not strongly dependent on $\mathcal{N}^{\text {ill }}$ for relatively small file sizes but for the largest file, the multi-threaded query shows better performance with optimally higher number of $\mathcal{N}^{\text {ill }}$. For small sizes of input files, the effective cost of query is dominated by the opening and closing of two files (e.g. $\mathcal{F}_{\text {in }} \& \mathcal{F}_{\text {idx }}$ ) as the cost of a seekg, call is nominal compared to file-io [43] costs.

From Fig. 5, it is clear that, data parsing gold standards like awk [44], [45], [46], sed [45], [47], [48], [49] however powerful, suffers from significantly longer access time compared to GSFRS. A potential integration of GSFRS with awk, sed would eventually turn these historically significant tools into very powerful and modern large scale data processing engines.

Simultaneous access to any random parts of the file is possible from arbitrarily large number of data processing units either through remote access or from processes on the same device. Also, the content of a given $\mathcal{F}_{\text {.idx }}$ can be trivially modified to higher or lower bit architectures if it is mathematically consistent to do so. Porting from completely different $O S$ architectures (e.g. Windows vs Linux) is as simple as copying the index file. Although, we have used newline as our record separator, it can be replaced by any other character separator specific to the need of a given data processing problem but without loss of generality. In future, we shall update the software to handle multiple characters as record separators as well as regular expression handlers. To preserve simplicity we allow only ASCII file but future development would generalize it to binary or other file types. From an algorithmic point of view, the query time should be independent of the file-size (or as independent as possible) however, from a hardware standpoint, there are certain limitations to this ideal scenario primarily due to the mechanism by which the seek ${ }_{8}^{8}$ function finds the address location in a block-by-block fashion.

Although, we developed the algorithm to address the large data file random access problem, it can be also be used for small data files as well particularly for expensive, complex calculations like $A C F, M S D$ etc. One of the immediate application area of this tool would be instant query embedded devices like fitbit [50], [51], MUSE [52], [53], wearable blood pressure monitor like Glabella [54] where intelligence is gathered from raw data samples collected through sensors within the device itselft. One easy way to use this tool is to generate the index file on-the-fly so that, subsequent access won't necessitate reading the file from start again. Often, in the research community data is generated by one group of people (or devices) and analyzed by various other groups under diverse contexts. In such scenarios, if the data is indexed right at the source, lot of computations (and energy) can be saved in the long run as we'll pay for only the accessed part of the data. We heavily utilized the lock based multi-threaded [55] builtin apis so that, the most computationally expensive part, indexing is done as fast as possible by optimally utilizing the available computational resources in a given computer. The choice of index file extension (see Sec. B) is arbitrary and can be trivially modified as per contextual processing need. Future development would include compression of the indices into binary files as well as random access to more granular level of data (e.g. words, matrix elements etc.). Existing computations using commercially available softwares like Matlab [56], [57], Mathematica [58], [59], Octave [60], [61] etc. would speed up significantly by interfacing with the GSFRS's get_sample function through interfacing tools generally available as a feature of the commercial softwares. The biggest advantage of interfacing with GSFRS would be to have concurrent view of various data sections would be available either from multi-threaded or multi process [62], [63] environments. In realistic scenarios, large files should be indexed overnight right after recording of raw data if on-the-fly indexing is not possible. One important advantage of using GSFRS is a stupendous gain of computational efficiency and energy footprint by completely eliminating the need for redundant queries. It is to be noted that, some of the most computationally expensive operations performed during typical big-data driven calculations are, cost of parsing and maintenance of continuously running live databases. Under GSFRS philosophy of index and parse, once the indices are created it can be used or copied to other machines anytime in future without having to maintain a live database. With this advantage, all the redundant computations are made completely obsolete consistent with the philosophy pay only for what you use.

\section{ACKNOWLEDGEMENT}

The authors acknowledge logistical support from the Brown Mindfulness Center, Department of Epidemiology and School of Public Health, Brown University. We are thankful for financial support from Fetzer Memorial Trust Foundation. Part of this research was conducted using computational resources and services at the Center for Computation and Visualization, Brown University.
7. in terms of computing time

8. https://stackoverflow.com/questions/64904236/c-seekgcomplexity 


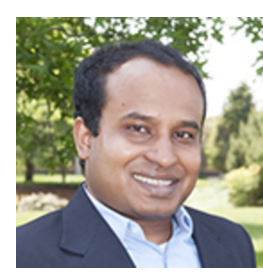

Prasanta Pal Prasanta Pal, PhD is an investigator of Epidemiology at the Brown Mindfulness Center, Brown School of Public Health. An applied physicist by training from Yale University School of Engineering, USA \& IIT Kharagpur, India, Dr. Pal has been the chief designer, director and developer of several neuro-feedback, datascience and mobile-application technologies directed towards human health and wellbeing. He is closely involved with bio-medical engineering research through first principle, minimal assumption methodos. Dr. Pal is deeply involved in building a suite of fundamental data science and medical imaging technologies to accelerate the field of data-driven diagnostic and healthcare interventions. Dr. Pal's work in medical technology is driven by the philosophy that mind and body works as one integrated system in a complimentary fashion. Holistic health outcomes are possible only when they are combined together through modern technology interfaces. He developed several technologies like MindView, MindScope etc. to help enhance various dimensions of human mind in a data driven, evidence based fashion. $\mathrm{He}$ serves in the editorial board of several biomedical engineering journals.

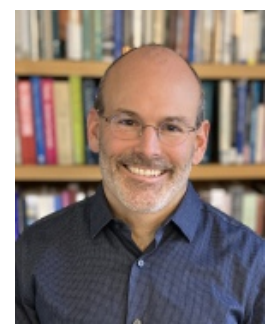

Judson A. Brewer Jud Brewer MD PhD is the Director of Research and Innovation at the Mindfulness Center and associate professor in Behavioral and Social Sciences at the School of Public Health and Psychiatry at the Medical School at Brown University. He also is a research affiliate at MIT. A psychiatrist and internationally known expert in mindfulness training for addictions, Brewer has developed and tested novel mindfulness programs for behavior change, including both in-person and app-based treatments for smoking, emotional eating, and anxiety. He has also studied the underlying neural mechanisms of mindfulness using standard and real-time fMRI, and source-estimated EEG, and is currently translating these findings into clinical use. $\mathrm{He}$ is the author of The Craving Mind: from cigarettes to smartphones to love, why we get hooked and how we can break bad habits (New Haven: Yale University Press, 2017) and the New York Times best-seller, Unwinding Anxiety: New Science Shows How to Break the Cycles of Worry and Fear to Heal Your Mind (Avery/Penguin Random House, 2021). Follow him on twitter @judbrewer.

\section{REFERENCES}

[1] A. S. Acharya, A. Prakash, P. Saxena, and A. Nigam, "Sampling: Why and how of it," Indian Journal of Medical Specialties, vol. 4, no. 2, pp. 330-333, 2013.

[2] J. Balcázar, Y. Dai, and O. Watanabe, "A random sampling technique for training support vector machines," in International Conference on Algorithmic Learning Theory. Springer, 2001, pp. 119-134.

[3] K. L. Clarkson and P. W. Shor, "Applications of random sampling in computational geometry, ii," Discrete E Computational Geometry, vol. 4, no. 5, pp. 387-421, 1989.

[4] C. Z. Mooney, Monte carlo simulation. Sage, 1997, no. 116.

[5] S. Raychaudhuri, "Introduction to monte carlo simulation," in 2008 Winter simulation conference. IEEE, 2008, pp. 91-100.

[6] K. Binder, D. Heermann, L. Roelofs, A. J. Mallinckrodt, and S. McKay, "Monte carlo simulation in statistical physics," Computers in Physics, vol. 7, no. 2, pp. 156-157, 1993.

[7] P. Pal, C. S. O'Hern, J. Blawzdziewicz, E. R. Dufresne, and R. Stinchcombe, "Minimal model for kinetic arrest," Physical Review E, vol. 78, no. 1, p. 011111, 2008.

[8] P. Pal, J. Blawzdziewicz, and C. S. O'Hern, "Quasi-one dimensional models for glassy dynamics," arXiv preprint arXiv:1401.0960, 2014.

[9] B. J. Berne, J. P. Boon, and S. A. Rice, "On the calculation of autocorrelation functions of dynamical variables," The Journal of Chemical Physics, vol. 45, no. 4, pp. 1086-1096, 1966.

[10] D. A. Griffith, "Spatial autocorrelation," A Primer (Washington, DC, Association of American Geographers), 1987.

[11] M. W. Woolrich, B. D. Ripley, M. Brady, and S. M. Smith, "Temporal autocorrelation in univariate linear modeling of fmri data," Neuroimage, vol. 14, no. 6, pp. 1370-1386, 2001.

[12] J. Kärger, "Straightforward derivation of the long-time limit of the mean-square displacement in one-dimensional diffusion," Physical Review A, vol. 45, no. 6, p. 4173, 1992.

[13] D. S. Grebenkov, "Time-averaged mean square displacement for switching diffusion," Physical Review E, vol. 99, no. 3, p. 032133, 2019.

[14] D. Geer, "Chip makers turn to multicore processors," Computer, vol. 38, no. 5, pp. 11-13, 2005.

[15] B. Schauer, "Multicore processors-a necessity," ProQuest discovery guides, pp. 1-14, 2008.

[16] P. F. Gorder, "Multicore processors for science and engineering," Computing in science $\mathcal{E}$ engineering, vol. 9, no. 2, pp. 3-7, 2007.

[17] A. Williams, C++ concurrency in action. Manning Publications, 2019, vol. 2.

[18] H.-J. Boehm and S. V. Adve, "Foundations of the c++ concurrency memory model," ACM SIGPLAN Notices, vol. 43, no. 6, pp. 68-78, 2008.

[19] G. Wiederhold, Database design. McGraw-Hill New York, 1983, vol. 1077.

[20] G. Powell, Beginning database design. John Wiley \& Sons, 2006.

[21] K. Chodorow, MongoDB: the definitive guide: powerful and scalable data storage. " O'Reilly Media, Inc.", 2013.

[22] C. Győrödi, R. Győrödi, G. Pecherle, and A. Olah, "A comparative study: Mongodb vs. mysql," in 2015 13th International Conference on Engineering of Modern Electric Systems (EMES). IEEE, 2015, pp. $1-6$.

[23] S. Papadopoulos, K. Datta, S. Madden, and T. Mattson, "The tiledb array data storage manager," Proceedings of the VLDB Endowment, vol. 10, no. 4, pp. 349-360, 2016.

[24] J. Bolewski and S. Papadopoulos, "Managing massive multidimensional array data with tiledb:-invited demo paper," in 2017 IEEE International Conference on Big Data (Big Data). IEEE, 2017, pp. 3175-3176.

[25] E. Greenfest-Allen, C. Klamann, O. Valladares, A. Kuzma, P. Gangadharan, Y. Y. Leung, C. J. Stoeckert, and L.-S. Wang, "Niagads alzheimer's genomicsdb: A resource for exploring alzheimer's disease genetic and genomic knowledge," bioRxiv, 2020.

[26] H. Li, "Big data technology accelerate genomics precision medicine," arXiv preprint arXiv:1701.09045, 2017.

[27] B. Stroustrup, A Tour of C++. Addison-Wesley Professional, 2018.

[28] M. Gregoire, Professional C++. John Wiley \& Sons, 2021.

[29] S. Meyers, Effektives modernes C++. O'Reilly Media, 2015.

[30] C. Kyriacou, P. Evripidou, and P. Trancoso, "Data-driven multithreading using conventional microprocessors," IEEE Transactions on Parallel and Distributed Systems, vol. 17, no. 10, pp. 1176-1188, 2006. 
[31] P. P. Gelsinger, "Microprocessors for the new millennium: Challenges, opportunities, and new frontiers," in 2001 IEEE International Solid-State Circuits Conference. Digest of Technical Papers. ISSCC (Cat. No. 01CH37177). IEEE, 2001, pp. 22-25.

[32] E. W. Weisstein, "Ceiling function," https://mathworld. wolfram. $\mathrm{com} /, 2002$.

[33] Y. Ling, T. Mullen, and X. Lin, "Analysis of optimal thread pool size," ACM SIGOPS Operating Systems Review, vol. 34, no. 2, pp. 42-55, 2000

[34] M. Posch, Mastering C++ Multithreading. Packt Publishing Ltd, 2017.

[35] J. Johansson, M. Gustafsson, and J. Delsing, "Ultra-low power transmit/receive asic for battery operated ultrasound measurement systems," Sensors and Actuators A: Physical, vol. 125, no. 2, pp. 317-328, 2006.

[36] P. W. Henderson, G. K. Lewis, N. Shaikh, A. Sohn, A. L. Weinstein, W. L. Olbricht, and J. A. Spector, "A portable high-intensity focused ultrasound device for noninvasive venous ablation," Journal of vascular surgery, vol. 51, no. 3, pp. 707-711, 2010.

[37] E. Shih, P. Bahl, and M. J. Sinclair, "Wake on wireless: An event driven energy saving strategy for battery operated devices," in Proceedings of the 8th annual international conference on Mobile computing and networking, 2002, pp. 160-171.

[38] X. Sun and N. Ansari, "Edgeiot: Mobile edge computing for the internet of things," IEEE Communications Magazine, vol. 54, no. 12, pp. 22-29, 2016.

[39] D. P. Miller, D. J. Atkinson, B. H. Wilcox, and A. H. Mishkin, “Autonomous navigation and control of a mars rover," in Automatic Control in Aerospace 1989. Elsevier, 1990, pp. 111-114.

[40] Y. Kuroda, K. Kondo, K. Nakamura, Y. Kunii, and T. Kubota, "Low power mobility system for micro planetary rover micro 5," in Artificial Intelligence, Robotics and Automation in Space, vol. 440, 1999 , p. 77.

[41] M. G. Harbour, "Programming real-time systems with $\mathrm{c} / \mathrm{c}++$ and posix," 2006.

[42] F. Salén, "Visualization of dynamic memory in c++ applications," 2019.

[43] B. Juneja, Programming with C++. New Age International, 2009.

[44] A. V. Aho, B. W. Kernighan, and P. J. Weinberger, "Awk-a pattern scanning and processing language," Software: Practice and Experience, vol. 9, no. 4, pp. 267-279, 1979.

[45] D. Dougherty and A. Robbins, sed E awk: UNIX Power Tools. " O'Reilly Media, Inc.", 1997.

[46] A. Robbins, Gawk: Effective AWK Programming. Free Software Foundation, 2004.

[47] B. Barnett, "Sed-an introduction and tutorial," 2008.

[48] M. Kerrisk, The Linux programming interface: a Linux and UNIX system programming handbook. No Starch Press, 2010.

[49] M. Welsh, M. K. Dalheimer, and L. Kaufman, Running Linux. O'Reilly \& Associates, Inc., 1999.

[50] K. Tsubouchi, R. Kawajiri, and M. Shimosaka, "Workingrelationship detection from fitbit sensor data," in Proceedings of the 2013 ACM conference on Pervasive and ubiquitous computing adjunct publication, 2013, pp. 115-118.

[51] M. Schellevis, B. Jacobs, C. Meijer, and J. de Ruiter, "Getting access to your own fitbit data," Radboud University, 2016.

[52] A. Przegalinska, L. Ciechanowski, M. Magnuski, and P. Gloor, "Muse headband: Measuring tool or a collaborative gadget?" in Collaborative Innovation Networks. Springer, 2018, pp. 93-101.

[53] O. E. Krigolson, C. C. Williams, A. Norton, C. D. Hassall, and F. L. Colino, "Choosing muse: Validation of a low-cost, portable eeg system for erp research," Frontiers in neuroscience, vol. 11, p. 109, 2017.

[54] C. Holz and E. J. Wang, "Glabella: Continuously sensing blood pressure behavior using an unobtrusive wearable device," Proceedings of the ACM on Interactive, Mobile, Wearable and Ubiquitous Technologies, vol. 1, no. 3, pp. 1-23, 2017.

[55] R. Rajwar and J. Goodman, "Transactional execution: Toward reliable, high-performance multithreading," IEEE Micro, vol. 23, no. 6, pp. 117-125, 2003.

[56] D. J. Higham and N. J. Higham, MATLAB guide. SIAM, 2016.

[57] T. A. Davis, MATLAB primer. CRC press, 2010.

[58] S. Wolfram et al., The MATHEMATICA® book, version 4. Cambridge university press, 1999.

[59] C. Rose and M. D. Smith, "Mathstatica: mathematical statistics with mathematica," in Compstat. Springer, 2002, pp. 437-442.
[60] J. W. Eaton, D. Bateman, S. Hauberg et al., Gnu octave. Network thoery London, 1997.

[61] J. S. Hansen, GNU Octave: Beginner's Guide: Become a Proficient Octave User by Learning this High-level Scientific Numerical Tool from the Ground Up. Packt Publishing Ltd, 2011.

[62] P. Calafiura, C. Leggett, R. Seuster, V. Tsulaia, P. Van Gemmeren et al., "Running atlas workloads within massively parallel distributed applications using athena multi-process framework (athenamp)," in Journal of Physics: Conference Series, vol. 664, no. 7. IOP Publishing, 2015, p. 072050.

[63] D. P. Bovet and M. Cesati, Understanding the Linux Kernel: from I/O ports to process management. "O'Reilly Media, Inc.", 2005.

[64] M. Batty, S. Owens, S. Sarkar, P. Sewell, and T. Weber, "Mathematizing c++ concurrency," ACM SIGPLAN Notices, vol. 46, no. 1, pp. 55-66, 2011.

[65] D. Griebler, J. Loff, G. Mencagli, M. Danelutto, and L. G. Fernandes, "Efficient nas benchmark kernels with c++ parallel programming," in 2018 26th Euromicro International Conference on Parallel, Distributed and Network-based Processing (PDP). IEEE, 2018, pp. 733-740. 
APPENDIX A

\section{SYMBOLS AND ACRONYMS}

1) GSFRS: Giant Signal File Random Sampler

2) $\mathcal{B}_{k}$ : Data-block $k$

3) $\mathcal{N}^{\mathcal{B}}$ : Number of data blocks used to divide the characters in the input file for processing with

4) $\mathcal{D}^{c}$ : Delimiter character

5) $\mathcal{D}_{k}^{c}: k^{t h}$ occurance of $\mathcal{D}^{c}$ in $\mathcal{F}_{\text {in }}$

6) $\mathcal{F}_{\text {in }}$ : Input data file

7) $\mathcal{N}^{\mathcal{F}_{\text {in }}}:$ Number of characters in $\mathcal{F}_{\text {in }}$

8) $\mathcal{F}_{\text {.idx }}:$ Index file

9) 墔: Threadpool

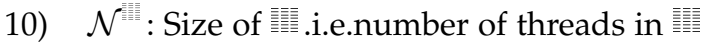

11) : Collection of hardware native threads available on the device

12) $\mathcal{N}^{\text {ii: }}$ : Number of hardware threads

13) m: Number of characters to represent a given index in $\mathcal{F}_{\text {.idx }}$

14) $\mathcal{I}_{k}^{c}$ : Index location of $\mathcal{D}_{k}{ }^{c}$

15) $\mathcal{N}^{\mathcal{I}_{k}^{c}}$ : Numerical value of the $k^{\text {th }}$ index located in $\mathcal{F}$.idx

16) $\quad N: O S$ architecture bits e.g. 64

\section{APPENDIX B MISC. INFORMATION}

The naming convention for $\mathcal{F}_{\text {.idx }}$ is such that the $\mathcal{F}_{\text {.idx }}$ name is same as $\mathcal{F}_{i n}$ with added extension suffix .idx 9 The file size of $\mathcal{F}_{\text {.idx }}$ can be further reduced by storing the indices in Hexadecimal format ${ }^{10}$. In situations where simultaneous access to either $\mathcal{F}_{i n}$ or $\mathcal{F}_{\text {.idx }}$, from different threads of the host $O S$ is necessary, a file-stream is opened specific to each thread using in-built $C++$ keyword thread_local. Although, we demonstrated the indexing process only for newline character, it can be easily extended to any other characters through a naming convention corresponding to each character. The information about the input files used for the numerical experiments are detailed in table 1. The source codebase is hosted at github ${ }^{11}$ Documentation and other helpful details are provide at a the hosting location ${ }^{12}$

TABLE 1: Information containing numer of lines, input file size, and index file size

\begin{tabular}{|c|c|c|c|c|}
\hline Serial & Data source & $\mathcal{N}^{\mathcal{D}^{c}}$ & $\mathcal{N}^{\mathcal{F}_{i n}}$ & $\mathcal{N}^{\mathcal{F}}$.idx \\
\hline 1 & BIOSEMI $E E G^{13}$ & 9963520 & $17 \mathrm{~GB}$ & $190 \mathrm{MB}$ \\
\hline 2 & SIMULATED $E E G$ & 10 & $18 \mathrm{~KB}$ & $262 \mathrm{~B}$ \\
\hline 3 & SIMULATED $E E G$ & 100 & $176 \mathrm{~KB}$ & $2 \mathrm{~KB}$ \\
\hline 4 & SIMULATED $E E G$ & 1000 & $1.7 \mathrm{MB}$ & $20 \mathrm{~KB}$ \\
\hline 5 & SIMULATED $E E G$ & 10000 & $17 \mathrm{MB}$ & $195 \mathrm{~KB}$ \\
\hline 6 & SIMULATED $E E G$ & 100000 & $172 \mathrm{MB}$ & $1.9 \mathrm{MB}$ \\
\hline 7 & SIMULATED $E E G$ & 1000000 & $1.7 \mathrm{~GB}$ & $19 \mathrm{MB}$ \\
\hline 8 & SIMULATED $E E G$ & 10000000 & $17 \mathrm{~GB}$ & $191 \mathrm{MB}$ \\
\hline
\end{tabular}

9. This choice is arbitrary

10. slated for a future version of the software

11. https://github.com/gsfrs/gsfrsbase

12. https://gsfrs.github.io

\section{APPENDIX C SOFTWARE STRUCTURE}

GSFRS is designed to be used as a standalone software as well as be integrated into other existing softwares by importing the .hpp header file. In its current version, the compiled executable would run from any standard terminal using simple command line options as described in Fig. 13 and more details are available in the software README.md file on the github repo. The code is entirely written in modern $\mathrm{C}++$ programming language and the command line options are facilitated by opensource project commandline ${ }^{14}$ interface The commandline interface is the entry point of

various operations that can be pereformed with the software. The most important option is ./GSFRS $-c \mathcal{F}_{\text {in }}$ to create an index of $\mathcal{F}_{i n}$. During the runtime of the index creation process each individual thread of 1 in in assigned the task of indexing a $\mathcal{B}_{k}$. After successful run of the index creation process, a new file with extension.$i d x$ would be generated at the location of $\mathcal{F}_{i n}$ containing all the indices. The other important options are $-p$ and $-r$ respectively. $-p$ is followed by the comma separated range structure sequence $n_{1}-n_{2}, n_{3}-n_{4}, n_{5}-n_{6} \ldots n_{7}-n_{8}$ etc. Each sample-range block $n_{k}-n_{l}$ means the parsing of all the samples between $n_{k} \&$ $n_{l}$ inclusive of the boundary points. Further details of the software would be made available on the github repository on the software documentation page.

\section{APPENDIX D CODE SAMPLES}

The function to get a single sample is described in Fig. 10 For multiple lines, a slightly modified version is demonstrated in Fig. 11 to facilitate a range query between a congiguous range of samples.

One of the most important function is the index_creation, as shown in Fig. 12 It performs the indexing and recording of indices on the disk. First, it creates a temporary file for each $\mathcal{B}_{k}$ and later concatenates them all to a single file $\mathcal{F}_{\text {.idx }}$ as the thread joining step ${ }^{15}$ If the call to this function is successful $\mathcal{F}_{\text {.idx }}$ is generated and made available to perform future query tasks. The temporary files containing indices for each $\mathcal{B}_{k}$ are removed from the disk when the indexing tasks, delegated to $\overline{\text { ini }}$ are successfully completed. Maintenance of the 讐 throughout the lifetime of the software, avoids the relatively expensive tasks of new thread creation and deletion. Smart-pointers are used whenever appropriate, to avoid unwanted memory leaks.

14. https://github.com/jarro2783/cxxopts

15. or equivalenty the reduce step [64], 65] in map-reduce framework 


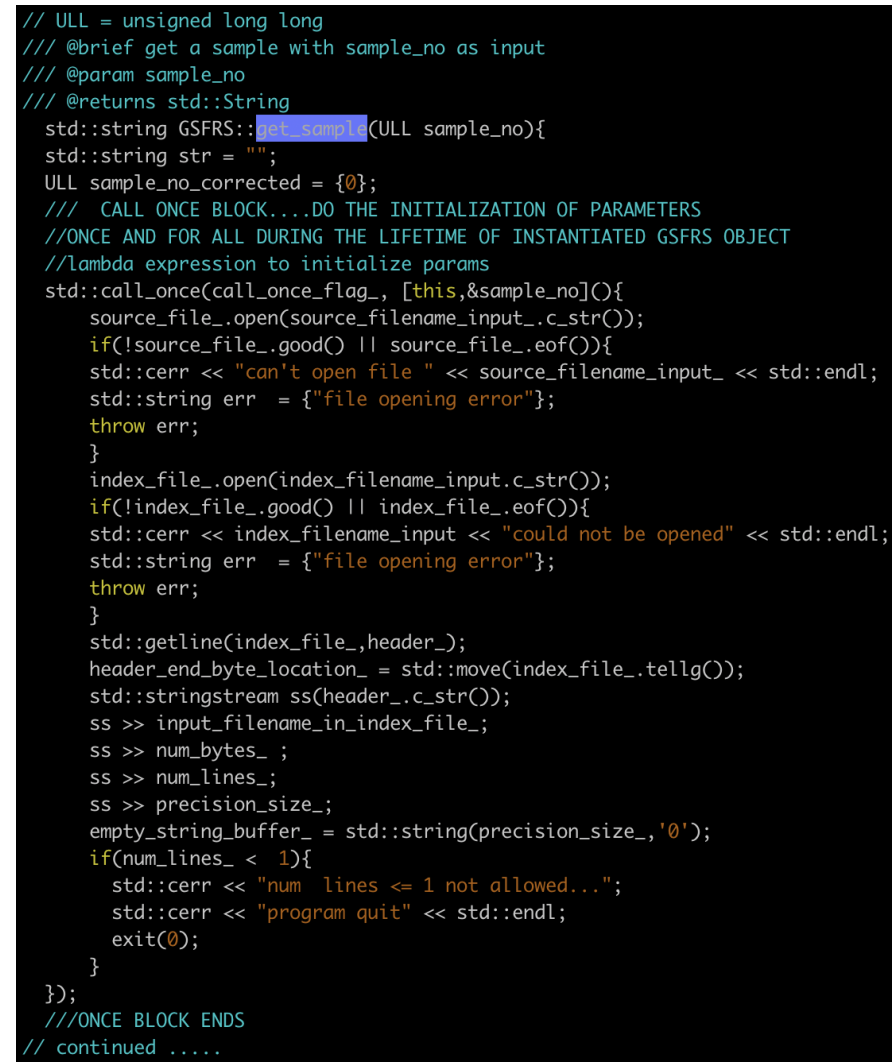

Fig. 10: The get_sample function prototype to fetch a single data sample based on the sample number query identifier

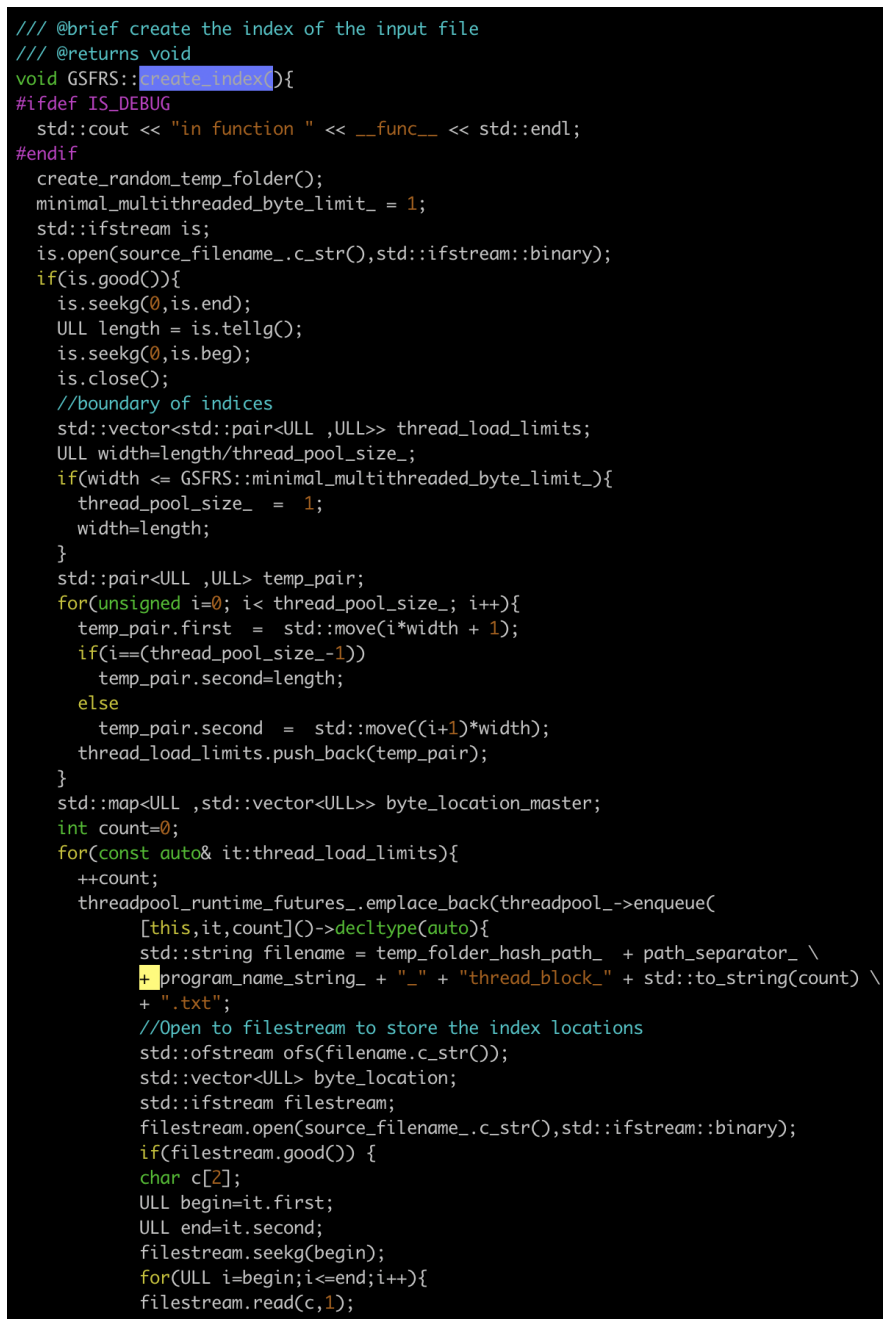

Fig. 12: The create_index function prototype to create the index file $\mathcal{F}_{\text {.idx }}$ from $\mathcal{F}_{\text {in }}$

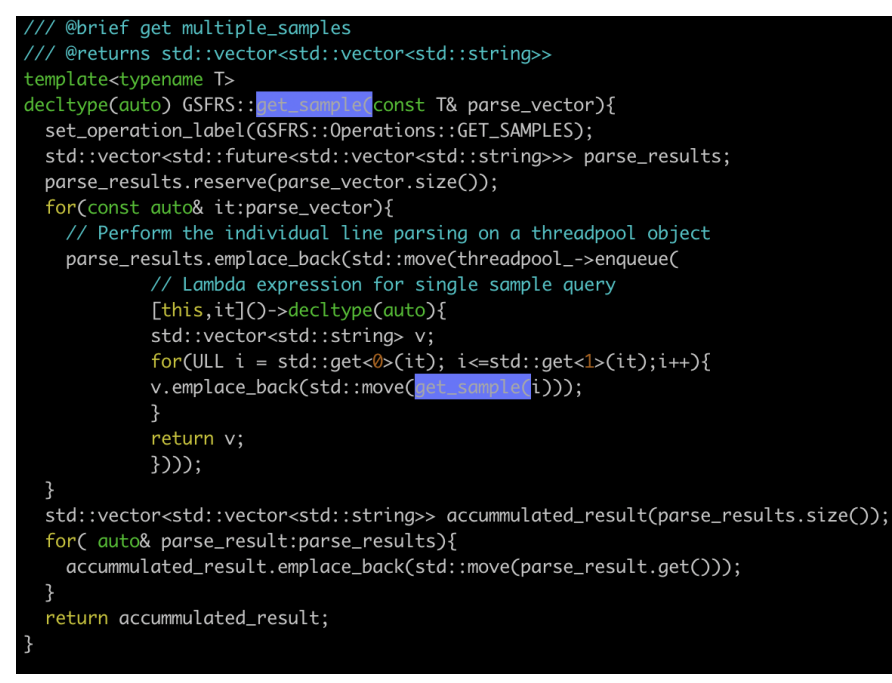

Fig. 11: The get_sample function prototype to get a multiple samples with a vector of sample numbers

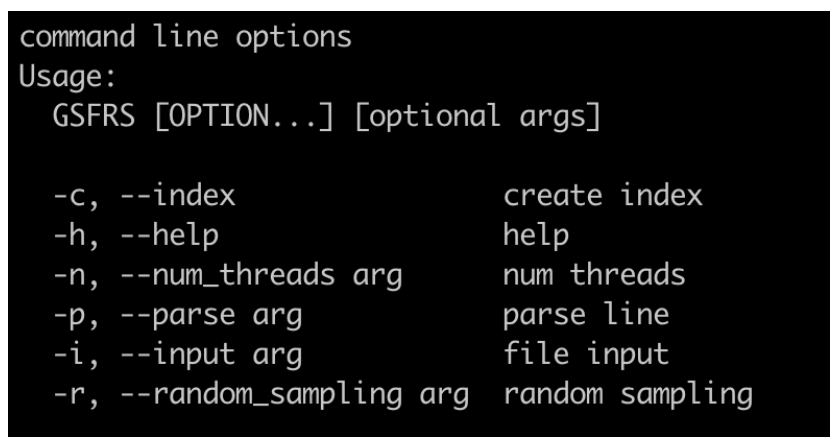

Fig. 13: Built-in command line options of the GSFRS software 